\title{
Improved Attainment through Outcome Based Education and a Case study
}

\author{
K.Pavani ${ }^{1}$, Associate Prof in ECE, VJIT \\ K. Vasanth Babu' ${ }^{2}$, HOD-ECE, VJIT \\ Y.Arun ${ }^{3}$, Assistant Prof in ECE, VJIT \\ K.Tarangini ${ }^{4}$, Associate Prof in ECE, VJIT \\ : ${ }^{1}$ pavanik@vjit.ac.in \\ : ${ }^{2}$ vkbece@vjit.ac.in \\ :3aruna@vjit.ac.in \\ : 4 tarangini.kuntla@gmail.com
}

and/or mentor based on the outcomes targeted. However the exiting Teaching-learning process creates a gap between university curriculum and students knowledge. Conducting regular classes only without any practical experiments and designs will not plays a good impact on student's results. The Program outcomes provides the information about ability of graduates to demonstrate knowledge in fundamentals of Basic Sciences, Humanities and Social Sciences, Engineering Sciences and apply the knowledge in professional core subjects, electives and projects. These outcomes enable the graduates to pursue higher studies and engage in $R \& D$ for a successful professional career. Based on CO-PO mapping, we can estimate effectiveness in teaching too. So finally with deep learning and analyzing, Outcome-Based Education reflects a great impact on student's results as well as in higher studies too. Control Systems Engineering is the one of the most important, interesting subject in electronics engineering stream.

\section{Traditional Teaching Approach and Literature Review:}

The traditional teaching-learning methodology is in the way of classroom teaching with chalk, board, OHP sheets and revisions. With Outcome Based Teaching-Learning, each subject specified by a course outcome (CO's), each of the CO's will addresses one or more program outcomes (PO's). Lizzie D'cruz Lecturer (Selection Grade), Department of Electronics and Communication Engineering, Dr. B. R. Ambedkar Institute of Technology, Port Blair, Andaman and Nicobar Islands, India presents $\mathrm{CO}, \mathrm{PO}$ attainment for VLSI Course for internal \& Board Exam results[1]. Course outcomes for digital switching systems will be defined and described by

Mark Ovinis proposed and described a Comparative Analysis of Attainment of Program Outcomes for Courses with and without the Use of Modern Tools and the usage of modern tools has led to slightly 
better attainment [2]. M.Rajendra Prasad developed project based teaching methodology for embedded engineering education to execute projects for better attainment [3]. Joni e. Spurlin presented a procedure to design better engineering education through assessment [4]; this book is written for engineering faculty and department chairs as a practical guide to improving the assessment processes for undergraduate and graduate engineering education in the service of improved student learning. M.Vasantha Lakshmi has defined outcome-based teaching process for microwave and radar [6]. This paper presents an Outcome Based Education and it is compared with traditional approach and attainment of the Course Outcomes (CO's) with Program Outcomes (PO'S).

\section{CO-PO Mapping in VJIT for R-15 Regulation:}

\section{Course: Control Systems Engineering \\ Course Code: A15415}

Course Outcome Statements indicating what a student can do after the successful completion of a course. At the end of the course the student should be able to get below points.

CO1: Demonstrate and understand the fundamentals of control systems.

CO2: Obtain the transient and steady state response of both linear and non-linear control systems.

C03: Determine and use models of physical systems in different forms suitable for use in the analysis and design of control systems.

CO4: Examine the stability of a closed-loop control system

CO5: Ability to represent and analyze control systems by state variable representation.

The program outcomes can be described what students are expected to know and would be able to do by the time of graduation. These relate to the skills, knowledge, and behaviors that students acquire as they progress through the program and those were shown in below table.1.

Table.1.The Program Outcomes (PO's)

\begin{tabular}{|c|c|c|c|}
\hline \multicolumn{4}{|c|}{ Program Outcome } \\
\hline PO1 & $\begin{array}{l}\text { Engineering } \\
\text { knowledge }\end{array}$ & PO 7 & $\begin{array}{l}\text { Environment and } \\
\text { sustainability }\end{array}$ \\
\hline PO 2 & Problem analysis & PO 8 & Ethics \\
\hline PO 3 & $\begin{array}{l}\text { Design / } \\
\text { development of } \\
\text { solutions }\end{array}$ & PO 9 & $\begin{array}{l}\text { Individual and team } \\
\text { work }\end{array}$ \\
\hline $\mathrm{PO} 4$ & $\begin{array}{l}\text { Conduct } \\
\text { investigations of } \\
\text { complex problems }\end{array}$ & PO10 & Communication \\
\hline PO 5 & Modern tool usage & PO11 & $\begin{array}{l}\text { Project management } \\
\text { and finance }\end{array}$ \\
\hline PO 6 & $\begin{array}{l}\text { The engineer and } \\
\text { society }\end{array}$ & PO12 & Life-long learning \\
\hline
\end{tabular}

\section{III.1.CO-PO Mapping for the course control system engineering:}

A sample CO-PO matrix for control systems engineering are given in below Table.2.Based on $\mathrm{CO}$ statements given. The CO-PO mapping has been done with correlation levels of 3,2 , and 1 . The notation of 3,2 and 1 denotes substantially (high), moderately (medium) and slightly (low).

\section{Table.2. CO-PO Mapping for A15415}

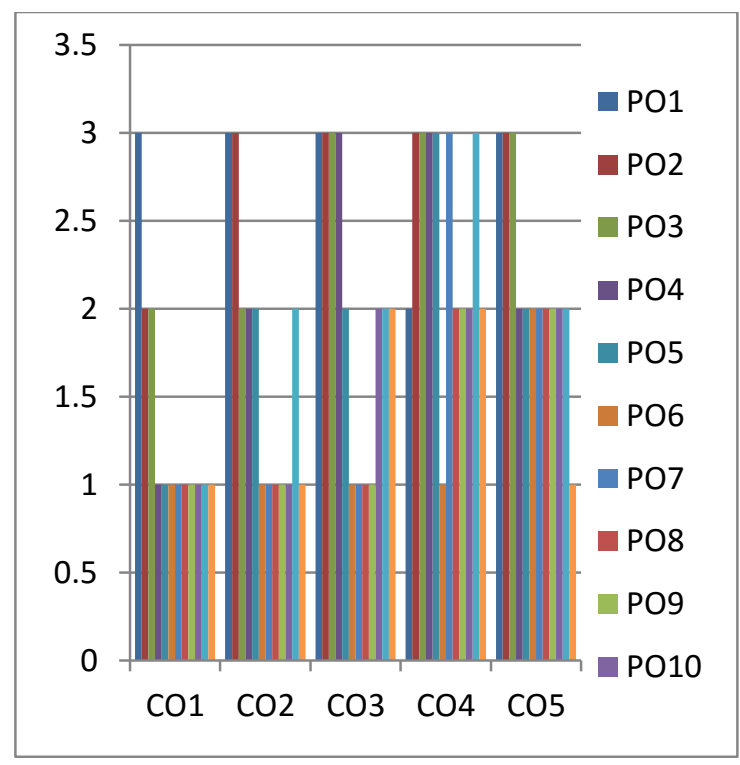

In VJIT, the students were assessed through the continuous internal exams and the end semester exams. The internal assessment includes descriptive tests and assignments. After two internal midterm exams, students may have a chance to write betterment exam to reduce absenteeism to improve their internal percentage. Out of three internal mid exams best of two will give the final internal performance of the student. If some components, to attain CO's/ PO's are not included in the curriculum provided by the affiliated university, then the institution makes additional efforts to impart such knowledge by covering aspects through "contents beyond syllabus" and "Gap Analysis". In Vidya Jyothi Institute Of Technology, the gap analysis is processed by taking feedback from the stake holders such as Employers, Alumni, Industry, Senior students, Faculty, Professional Bodies and Department Advisory Board (DAB).Based on the plan of action decided, the department arranges seminars, technical talks, workshops, training programs in Mat lab, NI-Lab view, Cisco Networking, ARM University program and industrial visits etc. Based on the student's 
performance, the $\mathrm{CO}-\mathrm{PO}$ attainments were calculated.

\section{Assessment of Course Outcomes:}

As per university guidelines $25 \%$ weight age is given to internal assessment and $75 \%$ weight age is given to external exam assessment. In VJIT Institute the overall attainment is calculated for every course by using direct method. The calculations for over all attainment are shown in below Table.3.

Table.3. Assessment of Course Outcomes

\begin{tabular}{|c|c|c|c|c|c|}
\hline $\begin{array}{l}\text { Course } \\
\text { out } \\
\text { Come }\end{array}$ & $\begin{array}{l}\text { Course } \\
\text { outcome } \\
\text { attainme } \\
\text { nt level } \\
\text { from } \\
\text { internal } \\
\text { assessme } \\
\text { nt }\end{array}$ & $\begin{array}{l}\text { Course } \\
\text { outcom } \\
\text { e } \\
\text { attainm } \\
\text { ent } \\
\text { level } \\
\text { from } \\
\text { univers } \\
\text { ity } \\
\text { exams }\end{array}$ & $\begin{array}{c}\mathrm{CO} \\
\text { Attain } \\
\text { ment }\end{array}$ & $\begin{array}{l}\text { Indirect } \\
\text { Attain } \\
\text { ment }\end{array}$ & $\begin{array}{l}\text { Over } \\
\text { all } \\
\text { Attain } \\
\text { ment }\end{array}$ \\
\hline $\begin{array}{l}\mathrm{CO} \\
\text { Attain } \\
\text { ment }\end{array}$ & $\begin{array}{l}\mathrm{a}_{1=} \\
\text { (Mid-1+ } \\
\text { Mid-2+ } \\
\text { Two } \\
\text { Assignm } \\
\text { ents) }\end{array}$ & $\mathrm{b}_{1}$ & $\begin{array}{l}\mathrm{c} 1= \\
(0.25 \\
\left(\mathrm{a}_{1}\right)+ \\
0.75 \\
\left.\left.\mathrm{~b}_{1}\right)\right)\end{array}$ & $\begin{array}{l}\mathrm{d} 1=((1 \\
* \mathrm{X}) \\
\frac{+(2 * Y)}{+(3 * \mathrm{Z}))} \\
\frac{(\mathrm{X}+\mathrm{Y}+}{\mathrm{Z})}\end{array}$ & $\begin{array}{l}0.8(\mathrm{c} 1) \\
+0.2(\mathrm{~d} 1 \\
)\end{array}$ \\
\hline
\end{tabular}

X---Number of students Opted for Low Option,

Y---Number of students Opted for Medium Option,

Z---Number of students Opteds.for Subsetantial High Option.

\section{Direct Assessment Evaluation:}

$$
1
$$

External Exams

Step1: Internal Examinations \&Assignments

The First midterm examination shall be conducted for 1-2.5 units of syllabus and second midterm examination shall be conducted for 2.5-5 units. 5 marks are allocated for Assignments (as specified by the concerned subject teacher) - first Assignment should be submitted before the conduction of the first mid, and the second Assignment should be submitted before the conduct of the second mid. The total marks secured by the student in each midterm examination are evaluated for 25 marks.

\section{Step 2: External Examinations}

\section{Total Duration: 3 Hours}

Total Marks: 75

Minimum Expected Marks for Pass: 26M (35\% of Maximum Marks 75)

Minimum Expected Marks for course Attainment: $45 \mathrm{M}(60 \%$ of Maximum Marks 75$)$

After completion of course by using defined Course Outcomes (CO's), attainment levels, the direct attainment will gives the information about $\mathrm{CO}$ attainment. For the academic year (2015-2019) the $\mathrm{CO}$ attainment is calculated for Control System Engineering course and that is shown in Table.4.

\section{Table.4.External Assessment}

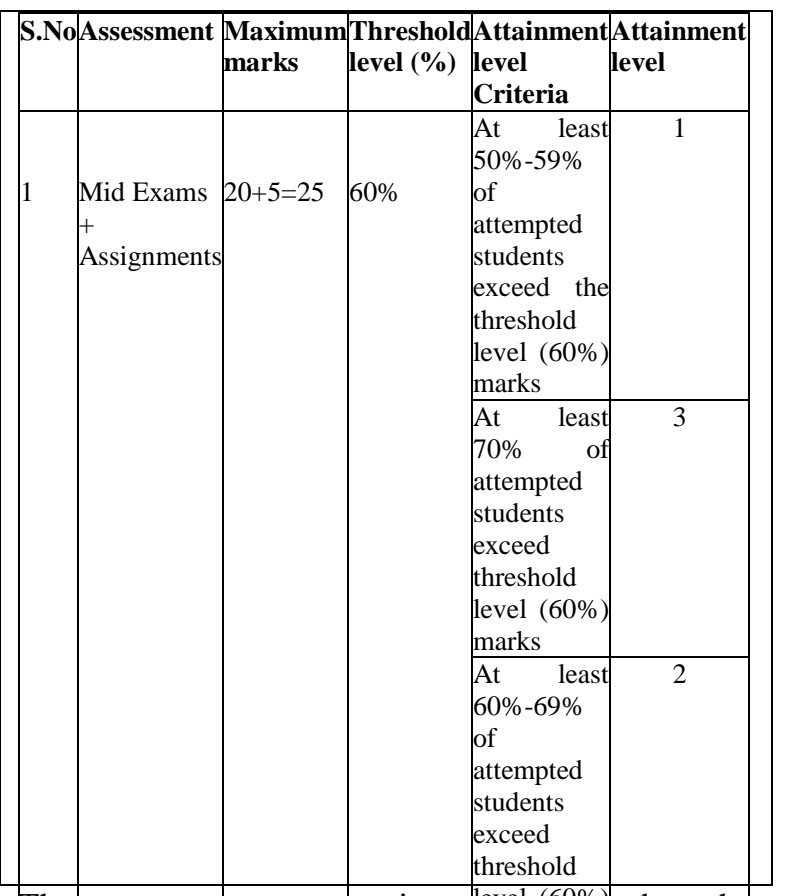

The course outcomes attainmehted dfinputed at the marks course Contro Threshold Attainment level Criteria shown in below Table.5.

Table.5.CO internal attainment At least 50\%-59\% of attempted students exceed the 75 assessment of co's torthe course at least $70 \%$ of attempted students exceed threshold

\begin{tabular}{|c|c|c|c|c|c|c|}
\hline co's & method & value & eavg & $\begin{array}{l}\text { Coley } \\
\text { attait } \\
\text { inter }\end{array}$ & 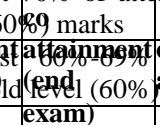 & $\begin{array}{l} \\
\text { oyerameschpt } \\
\text { attainment }\end{array}$ \\
\hline \multirow[t]{3}{*}{$\mathrm{col} 1, \mathrm{co}$} & $\begin{array}{l}\text { part-a } \\
\text { mid-i + } \\
\text { asm-i }\end{array}$ & 2.8 & 2.766 & \multirow{6}{*}{2.64} & \multirow{9}{*}{2.5} & \multirow{9}{*}{2.612} \\
\hline & \begin{tabular}{|l|} 
part- \\
bmid-i q1 \\
\end{tabular} & 2.7 & & & & \\
\hline & \begin{tabular}{|l|} 
Part-b \\
mid-i q2
\end{tabular} & 2.8 & & & & \\
\hline \multirow[t]{3}{*}{$\mathrm{co} 2, \mathrm{co} 3$} & $\begin{array}{l}\text { part-a } \\
\text { mid-i + } \\
\text { asm-i }\end{array}$ & 2.5 & \multirow[t]{3}{*}{2.7} & & & \\
\hline & \begin{tabular}{|l|} 
part-b \\
mid-i q3
\end{tabular} & 2.8 & & & & \\
\hline & \begin{tabular}{|l|} 
part-b \\
mid-i q4
\end{tabular} & 2.8 & & & & \\
\hline \multirow[t]{3}{*}{$\operatorname{co} 3, \operatorname{co} 4$} & $\begin{array}{l}\text { part-a } \\
\text { mid-ii + } \\
\text { asm-ii }\end{array}$ & 2.9 & \multirow[t]{3}{*}{2.533} & & & \\
\hline & \begin{tabular}{|l|} 
part-b \\
mid-ii q1
\end{tabular} & 2.2 & & & & \\
\hline & \begin{tabular}{|l} 
part-b \\
mid-ii q2
\end{tabular} & 2.5 & & & & \\
\hline
\end{tabular}




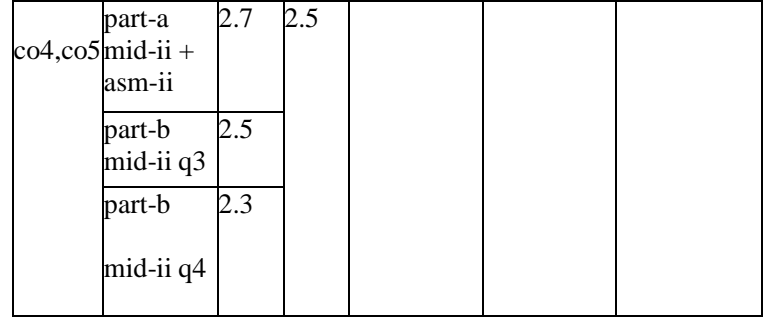

The improvement in attainment with teaching methodology under OBE after applying all those methods and after three certification programs students may gain best idea about their future plan as a Engineer and this paper is going to describe improved performance of one student, his academic profile, $\mathrm{CO}$ attainment, $\mathrm{PO}$ attainment, co-po attainmrnt with direct and indirect methods were shown in below Table.6. And the student name is Bharat Malaviya of 2015-2019 batches from ECE department in VJIT.

\section{Table.6.Academic Profile}

\begin{tabular}{|c|c|c|c|c|c|c|c|c|c|c|c|c|}
\hline & $\begin{array}{l}\text { Iarks } \\
\text { btained }\end{array}$ & & 5098 & $3 / 6350$ & & & GPA & & & 9.21 & & \\
\hline & $\begin{array}{l}\text { redits } \\
\text { btained }\end{array}$ & & $192 /$ & 192 & & & dbject & t Due & & 0/70 & & \\
\hline S & Code & subject & $\begin{array}{l}\text { Int } \\
\text { Ma }\end{array}$ & 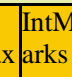 & \begin{tabular}{|l|l} 
& Ext \\
Max
\end{tabular} & $x^{\mid C h}$ & \begin{tabular}{l|l}
11 & To \\
1
\end{tabular} & ota $\mid \begin{array}{l}P_{c} \\
c\end{array}$ & $\begin{array}{c}\operatorname{Per} A \\
c \mid e\end{array}$ & $\begin{array}{l}\text { ActCr } \\
\text { edits }\end{array}$ & $\begin{array}{l}\text { Sta } \\
\text { us }\end{array}$ & $\begin{array}{l}\text { Gra } \\
\text { de }\end{array}$ \\
\hline & & \begin{tabular}{|l|} 
I/IV \\
SEM \\
\end{tabular} & & & & $\begin{array}{l}\mathrm{dec} \\
\mathrm{mb} \\
\end{array}$ & \begin{tabular}{l|l} 
ce & \\
eer
\end{tabular} & & & & & \\
\hline 1 & $\begin{array}{l}\text { A11 } \\
001\end{array}$ & $\begin{array}{l}\text { English- } \\
\text { I }\end{array}$ & $n-25$ & 23 & 75 & 59 & 82 & \begin{tabular}{l|l}
2 & 8 \\
& 0 \\
\end{tabular} & $\begin{array}{l}82.2 \\
00\end{array}$ & 2.00 & $\mathrm{P}$ & $\mathrm{O}$ \\
\hline 2 & \begin{tabular}{l|} 
A11 \\
002
\end{tabular} & $\begin{array}{l}\text { Mathem } \\
\text { atics - I }\end{array}$ & $\begin{array}{ll}\mathrm{m} & 25 \\
\end{array}$ & 25 & 75 & 64 & 89 & \begin{tabular}{l|l}
9 & 8 \\
& 0 \\
\end{tabular} & $\begin{array}{l}89.3 \\
00\end{array}$ & 3.00 & $\mathrm{P}$ & $\mathrm{O}$ \\
\hline 3 & \begin{tabular}{|l|} 
A11 \\
003 \\
\end{tabular} & $\begin{array}{l}\text { Enginee } \\
\text { ring } \\
\text { Physics- } \\
\text { I }\end{array}$ & 25 & 21 & 75 & 39 & 60 & 6 & 00.3 & 3.00 & $\mathrm{P}$ & A \\
\hline 4 & $\begin{array}{l}\text { A11 } \\
502\end{array}$ & \begin{tabular}{|l}
$\mathrm{C}$ \\
Program \\
ming -
\end{tabular} & $\mathrm{m}^{25}$ & 25 & 75 & 55 & 80 & 8 & 00.3 & 3.00 & $\mathrm{P}$ & $\mathrm{O}$ \\
\hline 5 & $\begin{array}{l}\text { A11 } \\
201\end{array}$ & $\begin{array}{l}\text { Electric } \\
\text { al } \\
\text { Circuits }\end{array}$ & $\begin{array}{ll}c & 25 \\
c & \end{array}$ & 18 & 75 & 48 & 66 & 6 & $\begin{array}{c}66.3 \\
00\end{array}$ & 3.00 & $P$ & A \\
\hline 6 & \begin{tabular}{|l|} 
A11 \\
004 \\
\end{tabular} & $\begin{array}{l}\text { Enginee } \\
\text { ring } \\
\text { Chemist } \\
\text { ry }\end{array}$ & $\mathrm{st} 25$ & 25 & 75 & 44 & 69 & 6 & 90.3 & 3.00 & $\mathrm{P}$ & A \\
\hline & esult (\% & $\%): 82.44$ & & & & & & & & & & \\
\hline & $e^{\text {Cod }}$ & subject & $\begin{array}{l}\text { IntM } \\
\text { ax }\end{array}$ & $\begin{array}{l}\text { IntM } \\
\text { arks }\end{array}$ & \begin{tabular}{|l|} 
Ext \\
Max
\end{tabular} & Ch1 & Ch2 & $\begin{array}{c}\text { To } \\
\text { tal }\end{array}$ & $\begin{array}{l}\text { Per } \\
c\end{array}$ & $\mid \begin{array}{l}\text { ActC } \\
\text { edits }\end{array}$ & $\begin{array}{l}\mathrm{Cr} \\
\mathrm{C}\end{array}$ & $\int_{\mathrm{ad}}^{\mathrm{Gr}} \mathrm{ad}$ \\
\hline & & $\begin{array}{l}\text { I/IV } \\
\text { SEM II } \\
\end{array}$ & & & & $\begin{array}{l}\text { may } \\
2016\end{array}$ & $\begin{array}{l}\text { nove } \\
\text { mber }\end{array}$ & & & & & \\
\hline & \begin{tabular}{l|l}
$\mathrm{A} 12$ & $\mathrm{E}$ \\
005
\end{tabular} & $\begin{array}{l}\text { English } \\
\text {-II }\end{array}$ & 25 & 24 & 75 & 51 & & 75 & $\begin{array}{l}75 . \\
00\end{array}$ & 2.00 & $\mathrm{P}$ & $\mathrm{A}+$ \\
\hline 2 & \begin{tabular}{|l|l|l|l|l} 
A12 & E \\
306 & C \\
& c \\
\end{tabular} & \begin{tabular}{|l|} 
Engine \\
ering \\
Graphi \\
cs \\
\end{tabular} & 25 & 25 & 75 & 38 & 38 & 63 & 63. & 3.00 & $\mathrm{P}$ & A \\
\hline 3 & \begin{tabular}{|l|l|l|l|l|l} 
A12 & e \\
007 & $\mathrm{~F}$ \\
\end{tabular} & \begin{tabular}{|l|} 
Engine \\
ering \\
Physics \\
-II \\
\end{tabular} & 25 & 25 & 75 & 47 & 57 & 82 & 82. & 3.00 & $\mathrm{P}$ & $\mathrm{O}$ \\
\hline 4 & \begin{tabular}{l|l}
$\mathrm{A} 12$ & $\mathrm{~N}$ \\
006 & $\mathrm{n}$ \\
\end{tabular} & \begin{tabular}{|l|} 
Mathe \\
matics
\end{tabular} & 25 & 25 & 75 & 50 & & 75 & $\begin{array}{l}75 . \\
00\end{array}$ & 3.00 & 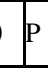 & $\mathrm{A}+$ \\
\hline
\end{tabular}

\begin{tabular}{|c|c|c|c|c|c|c|c|c|c|c|c|c|}
\hline & & - II & & & & & & & & & & \\
\hline 5 & $\begin{array}{l}\text { A12 } \\
009\end{array}$ & $\begin{array}{l}\text { Mathe } \\
\text { matics } \\
- \text { III }\end{array}$ & 25 & 25 & 75 & 43 & 75 & $\begin{array}{l}10 \\
0\end{array}$ & $\begin{array}{l}100 \\
.00\end{array}$ & 3.00 & $\mathrm{P}$ & $\mathrm{O}$ \\
\hline 6 & \begin{tabular}{|l|} 
A12 \\
503 \\
\end{tabular} & \begin{tabular}{|l}
$\mathrm{C}$ \\
Progra \\
mming \\
- II
\end{tabular} & 25 & 24 & 75 & 74 & & 98 & $\begin{array}{l}98 . \\
00\end{array}$ & 3.00 & $\mathrm{P}$ & $\mathrm{O}$ \\
\hline \multicolumn{4}{|c|}{ Result(\%):86.79 } & & & & & & & & & \\
\hline
\end{tabular}

\begin{tabular}{|l|l|l|l|l|l|l|l|l|l|l|l|}
\hline S. & Int & Ext & Tot & Per & ActCr & Stat Gra
\end{tabular}

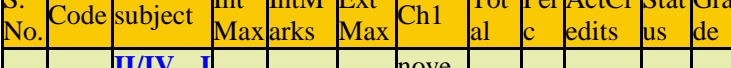

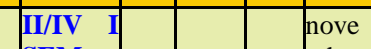

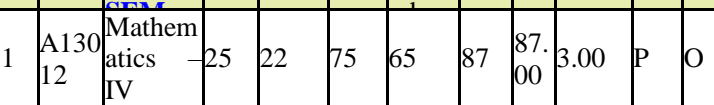

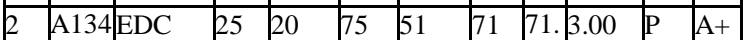

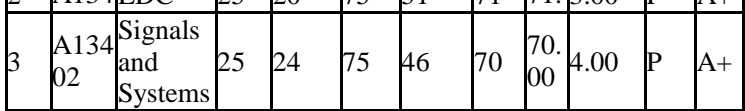
\begin{tabular}{|l|l|l|l|l|l|ll|l|l|l|}
\hline 4 & A134 & STLD & 25 & 23 & 75 & 49 & 72 & 72.3 .00 & P & A+ \\
\hline
\end{tabular} \begin{tabular}{|l|l|l|l|l|l|l|l|l|l|l|}
\hline 5 & A134 & EMI & 25 & 21 & 75 & 49 & 70 & 70.3 .00 & P & A+ \\
\hline 6 & A134 & TSSP & 25 & 24 & 75 & 41 & 65 & 65 & & \\
\hline
\end{tabular} \begin{tabular}{|l|l|l|l|l|l|l|l|l|l|l|}
\hline 6 & A134 & PTSP & 25 & 24 & 75 & 41 & 65 & 65.4 .00 & P & A \\
\hline
\end{tabular} Result(\%):76.80

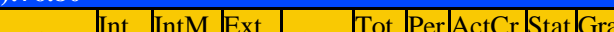

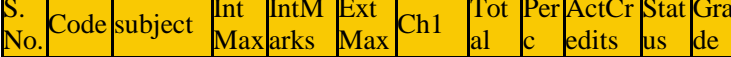

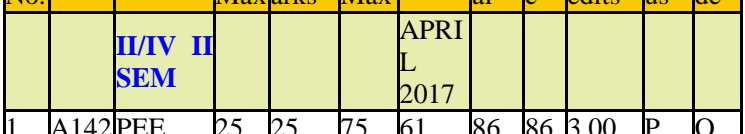

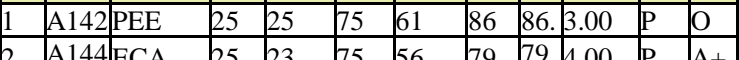

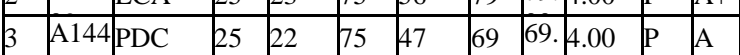

\begin{tabular}{|l|l|l|l|l|l|l|l|l|l|l|l|}
\hline 4 & A144 & EMTL & 25 & 24 & 75 & 49 & 73 & 73 & 4.00 & $\mathrm{P}$ & $\mathrm{A}+$ \\
\hline
\end{tabular}

\begin{tabular}{|l|l|l|l|l|l|l|l|l|l|l|l|}
\hline 5 & A144 & $\begin{array}{l}\text { Digital } \\
\text { System }\end{array}$ & 25 & 25 & 75 & 62 & 87 & 87. & 3.00 & P & 0 \\
\hline
\end{tabular}

\begin{tabular}{ll|l|l|l|l|l|l|l|l|l|l|l|}
5 & $\begin{array}{l}\text { A144 } \\
12\end{array}$ & System & 25 & 25 & 75 & 62 & 87 & 87. & 3.00 & $\mathrm{P}$ & $\mathrm{O}$
\end{tabular}

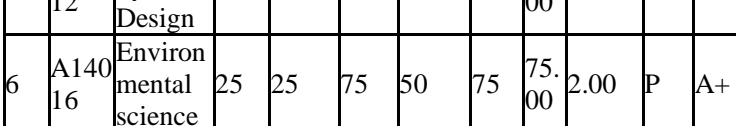

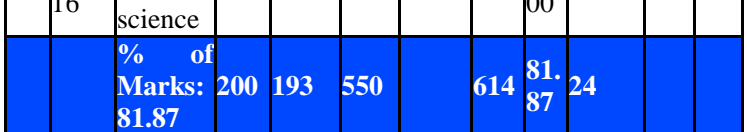

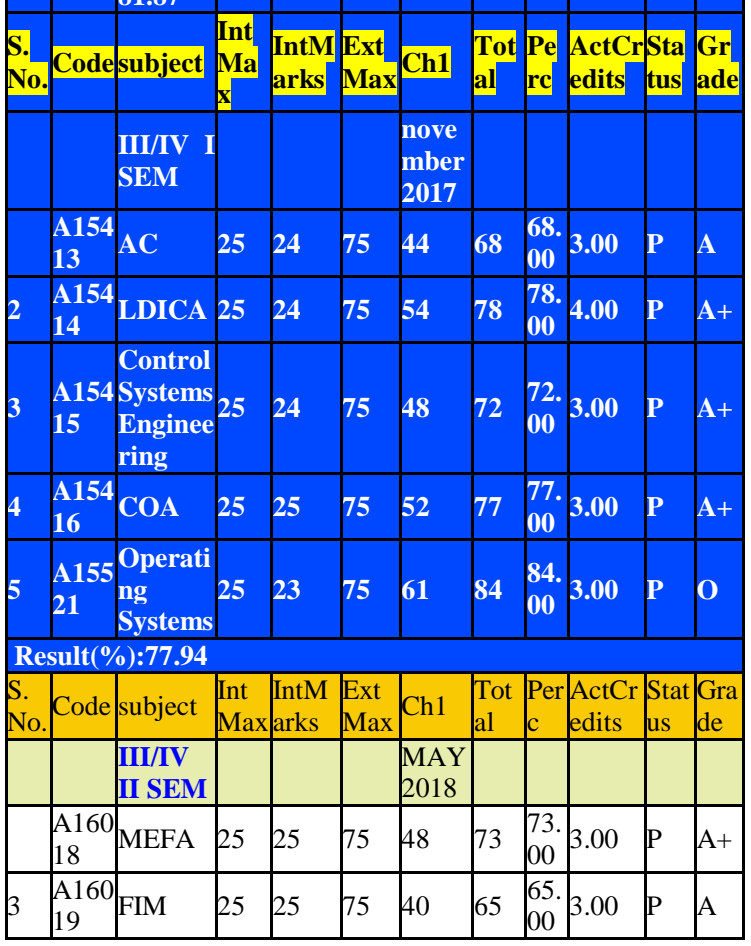




\begin{tabular}{|c|c|c|c|c|c|c|c|c|c|c|c|}
\hline 5 & $\begin{array}{l}\text { A164 } \\
22\end{array}$ & \begin{tabular}{|l|l} 
VLSI \\
Design
\end{tabular} & 25 & 24 & 75 & 56 & 80 & $\begin{array}{l}80 . \\
00\end{array}$ & 3.00 & $\mathrm{P}$ & $\mathrm{O}$ \\
\hline 6 & \begin{tabular}{|l|} 
A16 \\
TP1
\end{tabular} & PDBS & 25 & 22 & 75 & 55 & 77 & $\begin{array}{l}77 . \\
00\end{array}$ & 2.00 & $P$ & A+ \\
\hline 7 & \begin{tabular}{|l|l} 
A164 & \\
26 & 1 \\
\end{tabular} & $\begin{array}{l}\text { Program } \\
\text { ming in }\end{array}$ & 25 & 24 & 75 & 55 & 79 & $\begin{array}{l}79 . \\
00\end{array}$ & 3.00 & $P$ & A+ \\
\hline \multicolumn{12}{|c|}{ Result(\%):77.65 } \\
\hline $\begin{array}{l}\text { S. } \\
\text { No }\end{array}$ & Codes & subject & IntM & $\begin{array}{l}\text { IntM } \\
\text { arks }\end{array}$ & $\begin{array}{l}\text { Ext } \\
\text { Max }\end{array}$ & Ch1 & To & $\begin{array}{l}\text { Per } \\
c\end{array}$ & $\begin{array}{l}\text { ActCr } \\
\text { edits }\end{array}$ & $\begin{array}{l}\text { Sta } \\
\text { tus }\end{array}$ & $\begin{array}{l}\text { Gra } \\
\text { de }\end{array}$ \\
\hline & & $\begin{array}{l}\text { IV/IV } \\
\text { SEM }\end{array}$ & & & & $\begin{array}{l}\text { Nove } \\
\text { mber } \\
2018\end{array}$ & & & & & \\
\hline 3 & \begin{tabular}{|l|}
117 \\
433 \\
\end{tabular} & DIP & 25 & 24 & 75 & 41 & 65 & $\begin{array}{l}65 . \\
00\end{array}$ & 3.00 & $\mathrm{P}$ & A \\
\hline 4 & \begin{tabular}{|l|}
$\mathrm{A} 17$ \\
437
\end{tabular} & TSSN & 25 & 25 & 75 & 59 & 84 & \begin{tabular}{|l|}
84. \\
00
\end{tabular} & 3.00 & $\mathrm{P}$ & $\mathrm{O}$ \\
\hline 5 & \begin{tabular}{|l|l} 
A17 & \\
542 & I \\
\end{tabular} & $\begin{array}{l}\text { Web } \\
\text { Design }\end{array}$ & 25 & 23 & 75 & 44 & 67 & $\begin{array}{l}67 . \\
00 \\
\end{array}$ & 3.00 & $\mathrm{P}$ & $\mathrm{B}+$ \\
\hline 6 & \begin{tabular}{|l|l|} 
A17 \\
432
\end{tabular} & AWE & 25 & 24 & 75 & 34 & 58 & \begin{tabular}{|l|}
58. \\
00
\end{tabular} & 3.00 & P & $\mathrm{B}+$ \\
\hline 7 & \begin{tabular}{|l|l}
$\mathrm{A} 17$ & \\
430
\end{tabular} & DC & 25 & 23 & 75 & 51 & 74 & $\begin{array}{l}74 . \\
00\end{array}$ & 3.00 & $\mathrm{P}$ & $A+$ \\
\hline 8 & \begin{tabular}{|l|l|}
$\mathrm{A} 17$ & $\mathrm{E}$ \\
431 & $\mathrm{E}$ \\
\end{tabular} & ESD & 25 & 23 & 75 & 50 & 73 & $\begin{array}{ll}73 . \\
00 \\
\end{array}$ & 3.00 & $\mathrm{P}$ & $A+$ \\
\hline 9 & \begin{tabular}{|l|l} 
& $\mathrm{I}$ \\
$\mathrm{A} 17$ & $\mathrm{C}$ \\
$4 \mathrm{P} 1$ & $N$ \\
& $\mathrm{P}$
\end{tabular} & \begin{tabular}{|l|} 
Industry \\
Oriented \\
Mini- \\
Project
\end{tabular} & - & - & 50 & 49 & 49 & $\begin{array}{l}98 . \\
00\end{array}$ & 2.00 & $\mathrm{P}$ & $\mathrm{O}$ \\
\hline \multicolumn{12}{|c|}{ Result(\%):77.00 } \\
\hline $\begin{array}{l}\text { S.I } \\
\text { o. }\end{array}$ & $\mathrm{N} \mid$ Code & subject & \begin{tabular}{|l|} 
Int \\
Max \\
\end{tabular} & $\begin{array}{l}\text { IntM } \\
\text { arks }\end{array}$ & \begin{tabular}{|l|} 
Ext \\
Max \\
\end{tabular} & Ch1 & \begin{tabular}{|l|} 
To \\
tal
\end{tabular} & \begin{tabular}{|l} 
Per \\
c
\end{tabular} & $\begin{array}{l}\text { ActCr } \\
\text { edits }\end{array}$ & $\begin{array}{l}\text { Sta } \\
\text { tus }\end{array}$ & $\begin{array}{l}\text { Gra } \\
\text { de }\end{array}$ \\
\hline & & IV/IV II & & & & May & & & & & \\
\hline & \begin{tabular}{|l|} 
A184 \\
TS
\end{tabular} & $\begin{array}{l}\text { Technical } \\
\text { Seminar }\end{array}$ & & 46 & - & -- & 46 & $\begin{array}{l}92 . \\
00 \\
\end{array}$ & 2.00 & $\mathrm{P}$ & $\mathrm{O}$ \\
\hline 2 & $\begin{array}{l}\mathrm{A} 184 \\
44\end{array}$ & $\begin{array}{l}\text { Radar } \\
\text { Engineeri } \\
\text { ng }\end{array}$ & $i^{25}$ & 24 & 75 & 44 & 68 & $\begin{array}{l}68 . \\
00\end{array}$ & 3.00 & $\mathrm{P}$ & $A$ \\
\hline 3 & \begin{tabular}{|l|} 
A184 \\
P2
\end{tabular} & $\begin{array}{l}\text { Major } \\
\text { Project }\end{array}$ & 50 & 47 & 150 & 142 & $\begin{array}{l}18 \\
9 \\
\end{array}$ & $\begin{array}{l}94 . \\
50\end{array}$ & 11.00 & $\mathrm{P}$ & $\mathrm{O}$ \\
\hline 4 & $\begin{array}{l}\mathrm{A} 184 \\
\mathrm{CV}\end{array}$ & \begin{tabular}{|l|} 
Compreh \\
ensive \\
Viva \\
Voce
\end{tabular} & 100 & 97 & - & -- & 97 & $\begin{array}{l}97 . \\
00\end{array}$ & 2.00 & $P$ & $\mathrm{O}$ \\
\hline 5 & $\begin{array}{l}\text { A184 } \\
42\end{array}$ & $\mathrm{CMC}$ & 25 & 24 & 75 & 42 & 66 & $\begin{array}{l}66 . \\
00\end{array}$ & 3.00 & $P$ & $A$ \\
\hline 6 & $\begin{array}{l}\text { A184 } \\
43\end{array}$ & $\mathrm{DCN}$ & 25 & 24 & 75 & 41 & 65 & $\begin{array}{l}65 . \\
00\end{array}$ & 3.00 & $\mathrm{P}$ & $A$ \\
\hline
\end{tabular}

End Exam Result for the Course-CSE is displayed for only few members out of combined strength of regular 240 plus 45 lateral entry students, to show the improvement in the results and student Bharat barring Roll number of 15911A0464 got total 72 marks for 100 shown in below Table.7.

Table.7.Results of the Batch 2015-2019 for the course Control System Engineering

\begin{tabular}{|c|c|c|c|c|c|c|c|c|c|c|c|}
\hline & & $\begin{array}{l}\text { MATI } \\
\text { AB }\end{array}$ & & & & & & & & & \\
\hline 8 & \begin{tabular}{|l} 
A164 \\
24
\end{tabular} & MPM & c 25 & 22 & 75 & 46 & 68 & $\begin{array}{l}68 . \\
00\end{array}$ & 3.00 & $\mathrm{P}$ & A \\
\hline 9 & $\begin{array}{l}\text { A164 } \\
23\end{array}$ & DSP & 25 & 25 & 75 & 49 & 74 & $\begin{array}{l}74 . \\
00\end{array}$ & 3.00 & $\mathrm{P}$ & A+ \\
\hline \multicolumn{3}{|c|}{ Roll Number } & \multicolumn{3}{|c|}{ Internal } & \multicolumn{3}{|c|}{ External } & \multicolumn{3}{|c|}{ Total } \\
\hline \multicolumn{3}{|c|}{ 15911A0461 } & \multicolumn{3}{|c|}{11} & \multicolumn{3}{|c|}{19} & \multicolumn{3}{|c|}{30} \\
\hline \multicolumn{3}{|c|}{ 15911A0462 } & \multicolumn{3}{|l|}{15} & \multicolumn{3}{|l|}{40} & \multicolumn{3}{|l|}{55} \\
\hline \multicolumn{3}{|c|}{$15911 \mathrm{~A} 0463$} & \multicolumn{3}{|l|}{23} & \multicolumn{3}{|l|}{45} & \multicolumn{3}{|l|}{68} \\
\hline \multicolumn{3}{|c|}{$\begin{array}{l}\text { Bharat } \\
\text { Malaviya of } \\
\text { 15911A0464 }\end{array}$} & \multicolumn{3}{|l|}{24} & \multicolumn{3}{|l|}{48} & \multicolumn{3}{|l|}{72} \\
\hline \multicolumn{3}{|c|}{ 15911A0465 } & \multicolumn{3}{|l|}{20} & \multicolumn{3}{|l|}{50} & \multicolumn{3}{|l|}{70} \\
\hline \multicolumn{3}{|c|}{ 15911A0466 } & \multicolumn{3}{|l|}{18} & \multicolumn{3}{|l|}{42} & \multicolumn{3}{|l|}{60} \\
\hline \multicolumn{3}{|c|}{ 15911A0467 } & \multicolumn{3}{|l|}{10} & \multicolumn{3}{|l|}{40} & \multicolumn{3}{|l|}{50} \\
\hline
\end{tabular}

End Exam Result for the Course-CSE

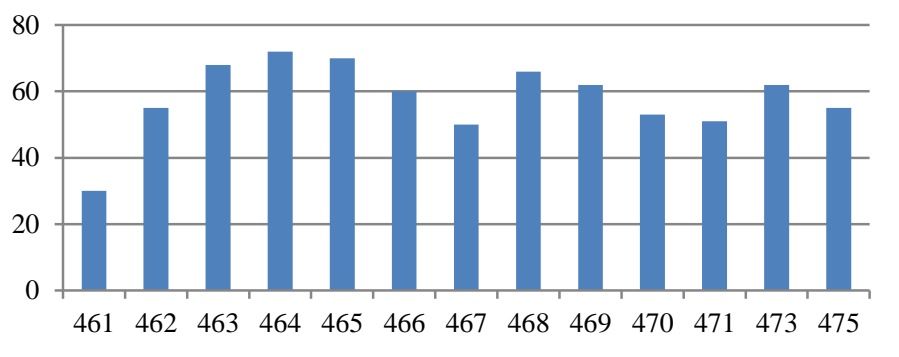

\begin{tabular}{l|l|l|l} 
15911A0468 & 17 & 49 & 66 \\
\hline
\end{tabular}

The results for the course shown below using chat to visulize that how many students were above the $60 \%$ of assigned threshold and how many members were below the $50 \%$ to complete the direct $\mathrm{CO}$ atainment,PO attainment and that is shown in below Figure.2.

Figure.2.End Exam Results for the course-CSE

VI. Course End Survey Form for the course CSE:

The Course End Survey Form for all subjects can be collected from students after successful completion of the B.Tech in VJIT and the respected faculty wil take care about that work. The course End Survey form for Control System Engineering is shown in below Table.8.

Table.8.Course End Survey From in VJIT 


\section{PO Attainment through direct Method:}

The PO Attainment of individual student through direct and indirect method can be evaluated after the completing their program. All these works have to be done under the guidance of Department Advisory Board $(\mathrm{DAB})$ in VJIT. In this regards The PO attainment for the student Bharath of roll number 15911A0464 is shown below Table.9.

TABLE.9. PO Attainment of the student Bharath of roll number 15911A0464

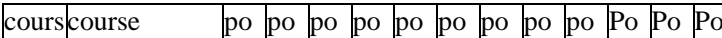
\begin{tabular}{|l|l|l|l|l|l|l|l|l|l|l|l|l}
$\mathrm{e}$ & 1 & 2 & 3 & 4 & 5 & 6 & 7 & 8 & 9 & 10 & 11 & 12 \\
\hline
\end{tabular} code

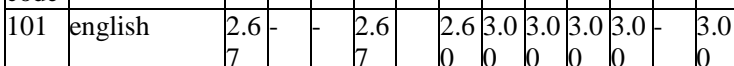

\begin{tabular}{|llllllllllllllllll}
\hline 102 & mathematics- & 3.0 & 3.0 & 3.0 & 3.0 & 2.0 & 3.0 & 2.0 & 2.0 & 2.0 & 3.0 & 1.0 & 3.0 \\
\hline
\end{tabular} \begin{tabular}{llllllllllllll}
1 & 0 & 0 & 0 & 0 & 0 & 0 & 0 & 0 & 0 & 0 & 0 & 0 \\
\hline
\end{tabular}

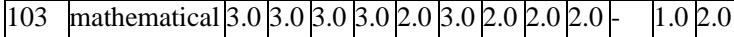
\begin{tabular}{l|llllllllll|lll} 
methods & 0 & 0 & 0 & 0 & 0 & 0 & 0 & 0 & 0 & & 0 & 0 \\
\hline
\end{tabular}

\begin{tabular}{ll|l|l|l|l|l|l|l|l|l|l|l|l}
104 & engineering & 3.0 & 2.8 & 2.4 & 2.8 & 2.0 & 2.0 & 1.6 & 2.0 & 1.0 & 1.0 & 1.0 & 2.0 \\
& 0 & 0 & 0 & 0
\end{tabular} \begin{tabular}{l|llllllllllllll} 
physics & 0 & 0 & 0 & 0 & 0 & 0 & 0 & 0 & 0 & 0 & 0 & 0 \\
\hline
\end{tabular}

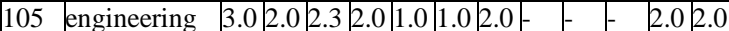
\begin{tabular}{llllllllll|l|ll} 
chemistry & 0 & 0 & 3 & 0 & 0 & 0 & 0 & & & 0 & 0
\end{tabular}

\begin{tabular}{llllllllllllllllllll}
\hline 106 & computer & 3.0 & 3.0 & 3.0 & 3.0 & 2.0 & 2.0 & 1.0 & 2.0 & 1.0 & 1.0 & 1.0 & 2.0 \\
\hline
\end{tabular} programming $0 \begin{array}{llllllllllllll}0 & 0 & 0 & 0 & 0 & 0 & 0 & 0 & 0 & 0 & 0 & 0\end{array}$

\begin{tabular}{|l|l|l|l|l|l|l|l|l|l|l|l|l|l|}
\hline 111 it & 3.0 & 3.0 & 2.0 & 1.0 & 1.0 & 1.0 & 2.0 & 2.0 & 2.0 & 2.0 & & 1.0 \\
\hline
\end{tabular} workshop/en $00 \begin{array}{lllllllllll} & 0 & 0 & 0 & 0 & 0 & 0 & 0 & 0 & 0 & 0\end{array}$ gg workshop

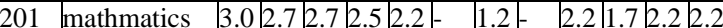
iii \begin{tabular}{lllll|l|l|l|l|l|l|l|}
0 & 5 & 5 & 0 & 5 & 5 & & 5 & 5 & 5 & 5 \\
\hline
\end{tabular} prpbability

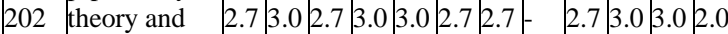
\begin{tabular}{l|l|l|l|l|l|l|l|l|l|lll} 
stochastic & 5 & 0 & 5 & 0 & 0 & 5 & 5 & & 5 & 0 & 0 & 0
\end{tabular} process

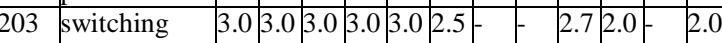
\begin{tabular}{|l|l|llllllll|l|l|l|l|l|} 
theory and & 0 & 0 & 0 & 0 & 0 & 0 & & & 5 & 0 & & 0
\end{tabular} logic design

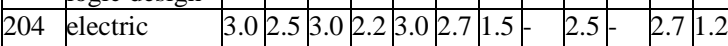
\begin{tabular}{l|l|l|l|l|l|l|l|l|l|l|l|l|} 
circuits & 0 & 0 & 0 & 5 & 0 & 5 & 0 & & 0 & & 5 & 5 \\
\hline
\end{tabular}

\begin{tabular}{ll|llllllllllllllllll}
205 & electronic & 2.2 & 3.0 & 3.0 & 2.7 & 3.0 & 2.7 & 2.7 & - & 3.0 & 3.0 & 3.0 & 2.0 \\
\hline
\end{tabular} \begin{tabular}{llllllllll|lllll} 
devices and & 5 & 0 & 0 & 5 & 0 & 5 & 5 & & 0 & 0 & 0 & 0
\end{tabular} circuits

\begin{tabular}{|l|l|l|l|l|l|l|l|l|l|l|l|l|l|l|l}
\hline 206 & signals and & 3.0 & 2.7 & 2.7 & 3.0 & 3.0 & 2.7 & 2.7 & - & 2.7 & 2.7 & 3.0 & 1.0 \\
\hline
\end{tabular} \begin{tabular}{llllllllllllllllll} 
systems & 0 & 5 & 5 & 0 & 0 & 5 & 5 & & 5 & 5 & 0 & 0 \\
\hline
\end{tabular}

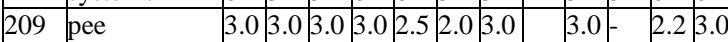

\begin{tabular}{|l|l|l|l|l|l|l|l|l|l|l|l|l|l|l|l|l}
\hline 210 & electronic & 3.0 & 3.0 & 3.0 & 3.0 & 3.0 & 2.7 & - & - & 3.0 & 3.0 & 3.0 & 3.0 \\
\hline
\end{tabular} \begin{tabular}{l|lllllll|l|llll} 
circuit & 0 & 0 & 0 & 0 & 0 & 5 & & 0 & 0 & 0 & 0
\end{tabular} analysis

211 pulse and $3.03 .03 .03 .03 .02 .7-0$ - 3.0 - 3.03 .0 \begin{tabular}{l|lllllll|l|ll} 
digital & 0 & 0 & 0 & 0 & 0 & 5 & 0 & 0 & 0
\end{tabular} circuits

$\begin{array}{llllllllllllllllll}212 & \text { environmenta } 3.0 & 3.0 & 3.0 & 3.0 & 2.0 & 3.0 & 3.0 & 3.0 & 3.0 & 3.0 & 3.0 & 3.0\end{array}$ \begin{tabular}{llllllllllllllll}
1 studies & 0 & 0 & 0 & 0 & 0 & 0 & 0 & 0 & 0 & 0 & 0 & 0 \\
\hline
\end{tabular}

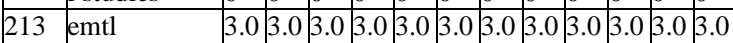

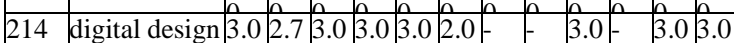
\begin{tabular}{llllllllllll|lll} 
through & 0 & 5 & 0 & 0 & 0 & 0 & & 0 & 0 & 0
\end{tabular} verilog hd

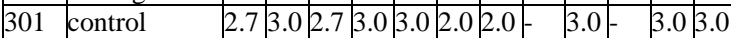
\begin{tabular}{l|l|l|l|l|l|l|l|l|l|l|l|l|} 
systems & 5 & 0 & 5 & 0 & 0 & 0 & 0 & 0 & 0 & 0 \\
\hline
\end{tabular} engineering

\begin{tabular}{lll|lllllllllll}
214 & digital design 3.0 & 2.7 & 3.0 & 3.0 & 3.0 & 2.0 & - & - & 3.0 & - & 3.0 & 3.0
\end{tabular}

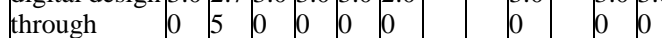
verilog hdl

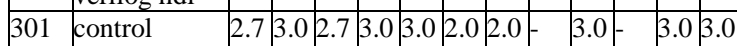
\begin{tabular}{l|lllllllll|l|l|lll} 
systems & 5 & 0 & 5 & 0 & 0 & 0 & 0 & & 0 & & 0 & 0
\end{tabular} engineering computer

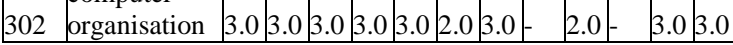

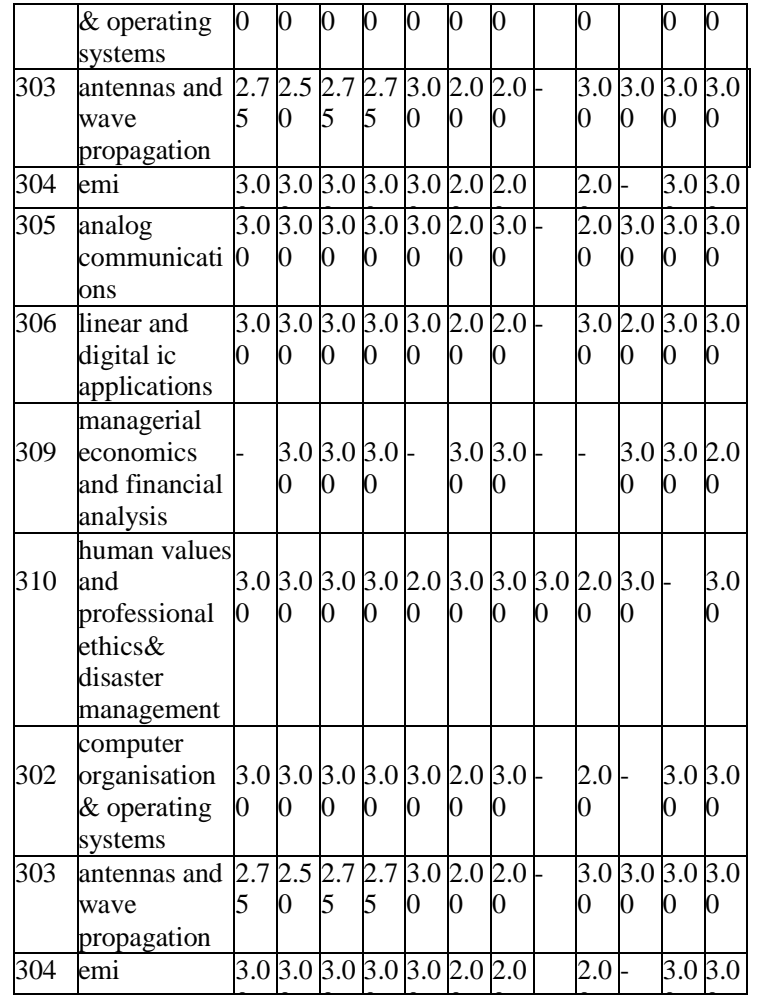

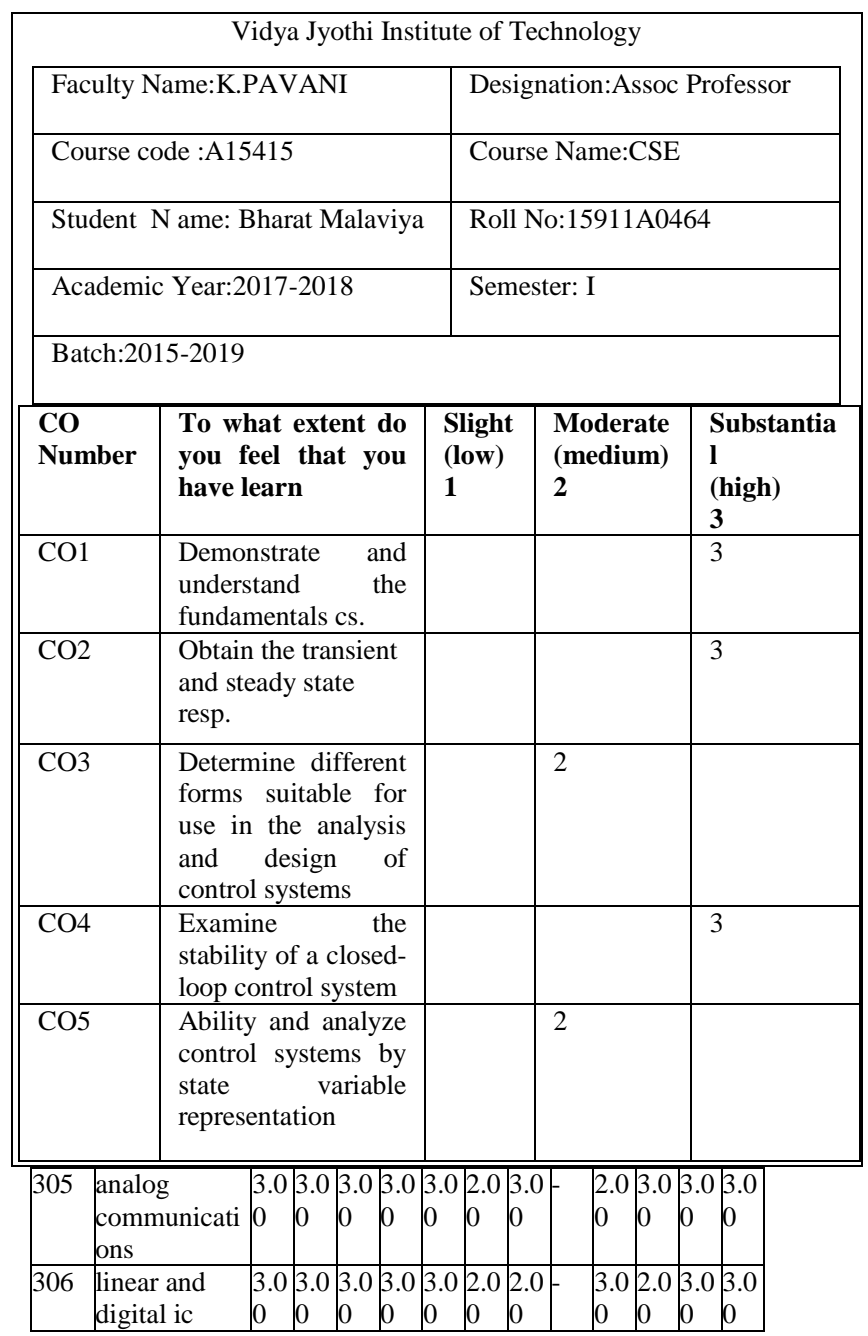




\begin{tabular}{|c|c|c|c|c|c|c|c|c|c|c|c|c|c|}
\hline & applications & & & & & & & & & & & & \\
\hline 309 & mefa & & 3.0. & 3.0 & 3.0 & & 3.0 & .00 & & & 3.0 & & 2.0 \\
\hline 310 & $\begin{array}{l}\text { human values } \\
\text { and }\end{array}$ & 3.0 & 3.0 & 3.0 & 3.0 & 2.0 & 3.0 & 3.0 & 3.0 & 2.0 & 3.0 & & \\
\hline & $\begin{array}{l}\text { professional } \\
\text { ethics\& } \\
\text { disaster } \\
\text { management }\end{array}$ & 0 & & 0 & 0 & 0 & 0 & 0 & 0 & 0 & 0 & & 0 \\
\hline 311 & digit & $3.0=$ & 3.0 & 3.0 & 3.0 & 3.0 & 2.0 & 2.0 & & 3.0 & 3.0 & 3.0 & 3.0 \\
\hline & $\begin{array}{l}\text { communicati } \\
\text { ons }\end{array}$ & 0 & & 0 & 0 & 0 & 0 & 0 & & 0 & 0 & 0 & 0 \\
\hline 312 & vlsi design & 2.5 & 2.5 & 3.0 & 3.0 & 3.0 & 2.5 & 2.5 & & 3.0 & 3.0 & 3.0 & 3.0 \\
\hline 313 & micro & 3.0 & 3.0 & 3.0 & 3.0 & 3.0 & 2.0 & 2.0 & 3.0 & 3.0 & 2.0 & 2.0 & 2.0 \\
\hline 314 & digital s & 3.0 & 3.0 & 3.0 & 3.0 & 3.0 & 3.0 & 3.0 & & 2.0 & 3.0 & 2.0 & 3.0 \\
\hline & $\mathrm{pr}$ & 0 & 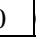 & 0 & 0 & 0 & 0 & 0 & & ) & & 0 & 0 \\
\hline & rage of & 2.9 & 2.8 & 2.8 & 2.8 & 2.5 & 2.2 & 2.2 & & & & & 2.4 \\
\hline & $\begin{array}{l}\text { direct } \\
\text { attainment }\end{array}$ & 4 & & 9 & 5 & 5 & 5 & 6 & 0 & & & 0 & 4 \\
\hline
\end{tabular}

Table.10.PO Direct Attainment results of Bharath of roll number 15911A0464:

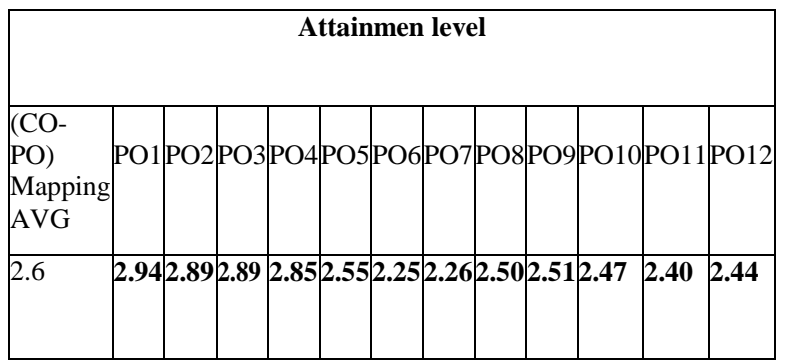

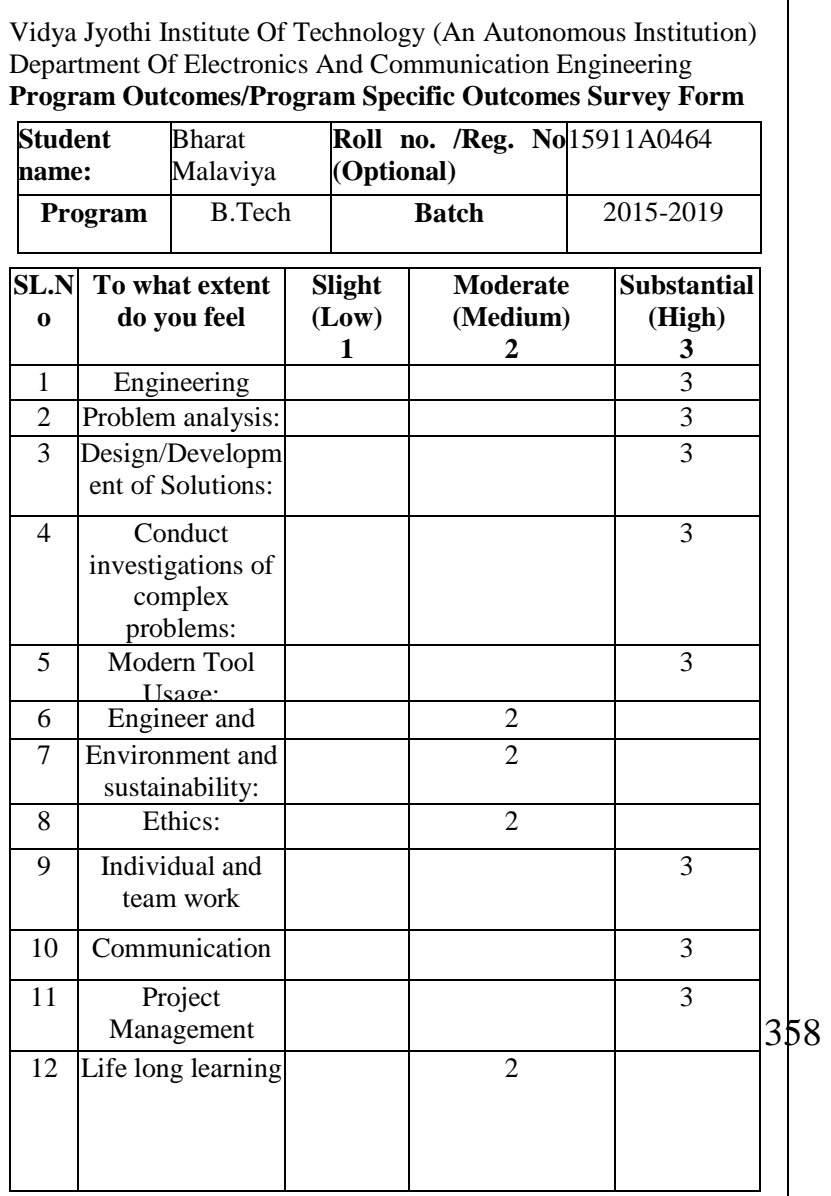

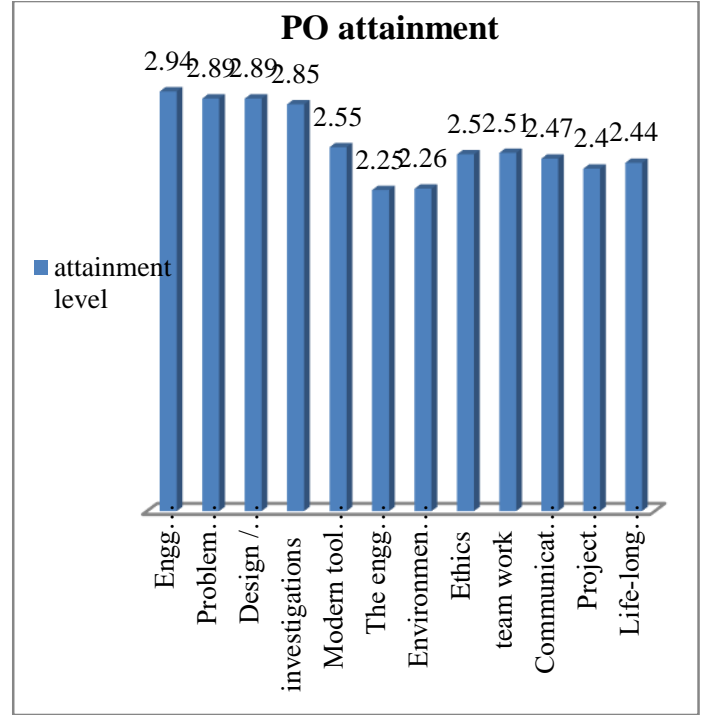

VIII.PO Attainment results through indirect method:

VIII.1.Analysis of Exit Survey Data:

Analysis of Exit survey data was carried out through questionnaire form in VJIT has collected after completion of final $8^{\text {th }}$ semester.Out of the

\section{Table.11.Program Outcome Survey Form}

$20 \%$ weightage allotted for indirect method, $10 \%$ is taken from the Exit survey. 5\% weight age allotted for Faculty survey and 5\% for Course completion survey. The course end survey for the student Bharat is already shown in chapter VI and results also presented.Now the Program outcome using exit survey data in VJIT is shown in below Table.11.and in table11.1.

\section{Table.11.1.Program Outcome Survey Form}

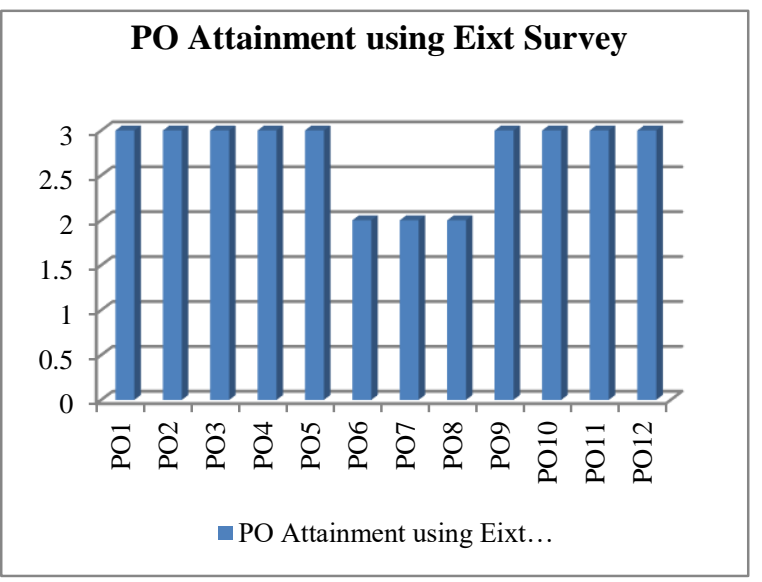

\section{IX.Conclusion:}

This paper presents complete $\mathrm{CO}$ attainment and $\mathrm{PO}$ Attainment of the course Control Systems 
Engineering and the course B.Tech-ECE of the student M.Bharath of Roll No 15911A0464 for the both internal \& Board Exam results using various surveys by using indirect and direct methodds. Study reveals that although result was $97 \%$ for the course CSE, the attainment was less for CO3 \& CO5. This analysis will help the faculty to plan new strategy for delivery, assessment and students involvement is also more important to improve practical knoweldge.In Vidya Jyothi Institute of Technology, students can able to access, E- Journals (IEEE explore), SONET CD'S for core subjects like EDC, COMMUNICATINS (it includes both AC and DC), STLD, MPMC and MICROWAVE ENGNNEERING, and Students are encouraging with Project based learning and Eletronics and communication Engg department is organizing many training programs for students like $\mathrm{Ni} \mathrm{Lab}$ view,ARM university program,Modelsim,Machine Learning with Mous and in VJIT placement and training programs were running in semester breaks to provide placement for Students in both software and core side.For that reason only Mr.Bharath attainments shows improvements compared to traditional methods.

\section{References:}

[1] Lizzie D'cruz Lecturer (Selection Grade), Department of Electronics and Communication Engineering, Dr. B. R. Ambedkar Institute of Technology, Port Blair, Andaman and Nicobar Islands, India: Transition from Traditional to Outcome Based Education- A Case Study. IOSR Journal of Research \& Method in Education (IOSRJRME) e-ISSN: 2320-7388,p-ISSN: 2320-737X Volume 7, Issue 4 Ver. I (Jul - Aug 2017), PP 38-45 www.iosrjournals.org

[2] Mark Ovinis ,Universiti Teknologi PETRONAS, Malaysia proposed A Comparative Analysis of Attainment of Program Outcomes for Courses with and without the Use of Modern Tools. 10.1051/matecconf/201822506022UTP-UMP-VIT

[3] M.Rajendra Prasad developed project based teaching methodology for embedded engineering education to execute projects for better attainment.Journal of Engineering Education Transformations, Volume 30, No. 3, January 2017, ISSN 2349-2473, eISSN 2394-1707.

[4]M Rajendra Prasad, D Krishna Reddy, Computer Based Teaching Methodology for Outcome-Based Engineering Education, 6th IEEE International Conference in Advanced Computing (IACC-IEEE Xplore), 2016.

[5]Designing Better Engineering Education through Assessment: A Practical Resource for Faculty and Department Chairs on Using Assessment and ABET Criteria to Improve Student Learning by Joni Spurlin (Editor), Sarah $\underline{\text { Rajala (Editor), Jerome P. Lavelle (Editor), Richard }}$ M. Felder (Foreword)

[6] RajeshwariHegde, "Implementing outcome based education for microcontrollers", MOOC, Innovation and Technology in Education (MITE), 2014 IEEE International Conference.

[7] M. Vasantha Lakshmi, "Outcome-Based Teaching: Microwave and Radar" IEEE International Conference on MOOC, Innovation and Technology in Education-MITE 2014. 